\title{
Atom-by-Atom Assembly in Aberration Corrected STEM and the Role of Chemistry at the Surface of Graphene
}

\author{
Ondrej Dyck, ${ }^{1,2}$ Songkil Kim, ${ }^{3}$ Elisa Jimenez-Izal, ${ }^{4,5}$ Anastassia N. Alexandrova, ${ }^{5,6}$ Sergei V. Kalinin ${ }^{1,2}$ \\ and Stephen Jesse ${ }^{1,2}$ \\ 1. Center for Nanophase Materials Science, Oak Ridge National Laboratory, Oak Ridge, TN. \\ 2. Institute for Functional Imaging of Materials, Oak Ridge National Laboratory, Oak Ridge, TN. \\ 3. Department of Mechanical Engineering, Pusan National University, Republic of Korea. \\ 4. Department of Chemistry and Biochemistry, University of California, Los Angeles, Los Angeles, CA. \\ 5. Kimika Fakultatea, Euskal Herriko Unibertsitatea (UPV/EHU), and Donostia International Physics \\ Center (DIPC), Donostia, Euskadi, Spain. \\ 6. California NanoSystems Institute, Los Angeles, CA.
}

In recent years many electron beam-induced sample changes have been documented in the (scanning) transmission electron microscope ((S)TEM), including catalytic etching, [1, 2] nanotube growth,[3] electron beam welding,[4] and controlled single atom movement.[5, 6] In order to bring these beaminduced changes under our control and enable wide ranging nanoscale fabrication techniques in the (S)TEM, a greater understanding of the surface chemistry of samples is needed. In the case of graphene, particular attention must be turned toward sample transfer and pretreatment to obtain favorable conditions in the microscope for experimentation in this regard. Here, we focus on exploring beam-induced chemical reactions with defects implanted in graphene.

An $\mathrm{Ar} / \mathrm{O}_{2}(90 \% / 10 \%)$ annealing protocol $\left(500{ }^{\circ} \mathrm{C}\right.$ for $\left.1.5 \mathrm{hrs}\right)$ was used which chemically alters the residues left from sample transfer and provides favorable imaging and atomic-scale experimentation environments. Figure 1 a)-c) illustrates what happens upon irradiation in the STEM. We observe immediate and significant contraction of the surface contamination exposing large areas of pristine graphene without a significant propensity to deposit hydrocarbon contamination. Once these clean areas are opened, dopant atoms may be introduced from the contaminant material, as illustrated in d)-f), by exposing an area of graphene to a $100 \mathrm{keV}$ probe and subsequently sputtering the contamination into the created defect. In these examples the dopant atoms are Si. Once dopant atoms have been introduced, the possibility of documenting surface chemical reactions at the defect site on the atomic level become possible. In particular, we are interested in controlled and reproducible chemical reactions which will be an enabling factor in the nanoscale fabrication paradigm in STEM. In g)-i) we observe a Si dimer undergoing a beam-induced chemical reaction with a $3^{\text {rd }} \mathrm{Si}$ atom where the Si replaces two $\mathrm{C}$ atoms and forms a Si rotor in the graphene lattice. This occurs due to the beam induced agitation of loosly bonded surface adatoms which randomly migrate under irradiation until they find a defect and bond more strongly. Following this model of attaching atoms to a defect site, the beam was scanned over a wide area and inspired the attachment of a $4^{\text {th }} \mathrm{Si}$ atom to the rotor, shown in j). Both structures are rotatable with the beam and irradiating this $4^{\text {th }}$ atom with the beam causes its ejection from the structure, $\mathrm{k}$ ) and 1). Single atom movement is also possible, and we illustrate the assembly of a Si dimer in $\mathrm{m}$ )-o), where the motion of the Si dopants through the lattice is tracked by the dotted lines. The inset in o) shows an atomic model of the final structure.

Finally, in p)-u), we show an image sequence of a dynamic chemical reaction between a Si defect cluster in a lightly contaminating environment (i.e. the presence of a volatile carbon containing gas). This 
contamination does not produce the typical amorphous carbon deposits in this case, but rather, results in the replacement of the $\mathrm{Si}$ dopants with $\mathrm{C}$, the healing of the lattice, and graphitic growth along the edge of the contaminated area. The concentration of hydrocarbon gas may be directly controllable through the use of heating stages and may significantly expand the available source materials through intentional introduction of various precursor materials designed to outgas and deposit or react with engineered defects.

References:

[1] H. Wang et al, Scientific Reports. 2 (2012), p. 995.

[2] W.L. Wang et al, Nano Letters. 14 (2014), p. 450.

[3] I.G. Gonzalez-Martinez et al, Nano Letters. 14 (2014), p. 799.

[4] M. Terrones et al, Physical Review Letters. 89 (2002), p. 075505.

[5] T. Susi et al, 2D Materials. 4 (2017), p. 042004.

[6] O. Dyck et al, Applied Physics Letters. 111 (2017), p. 113104.
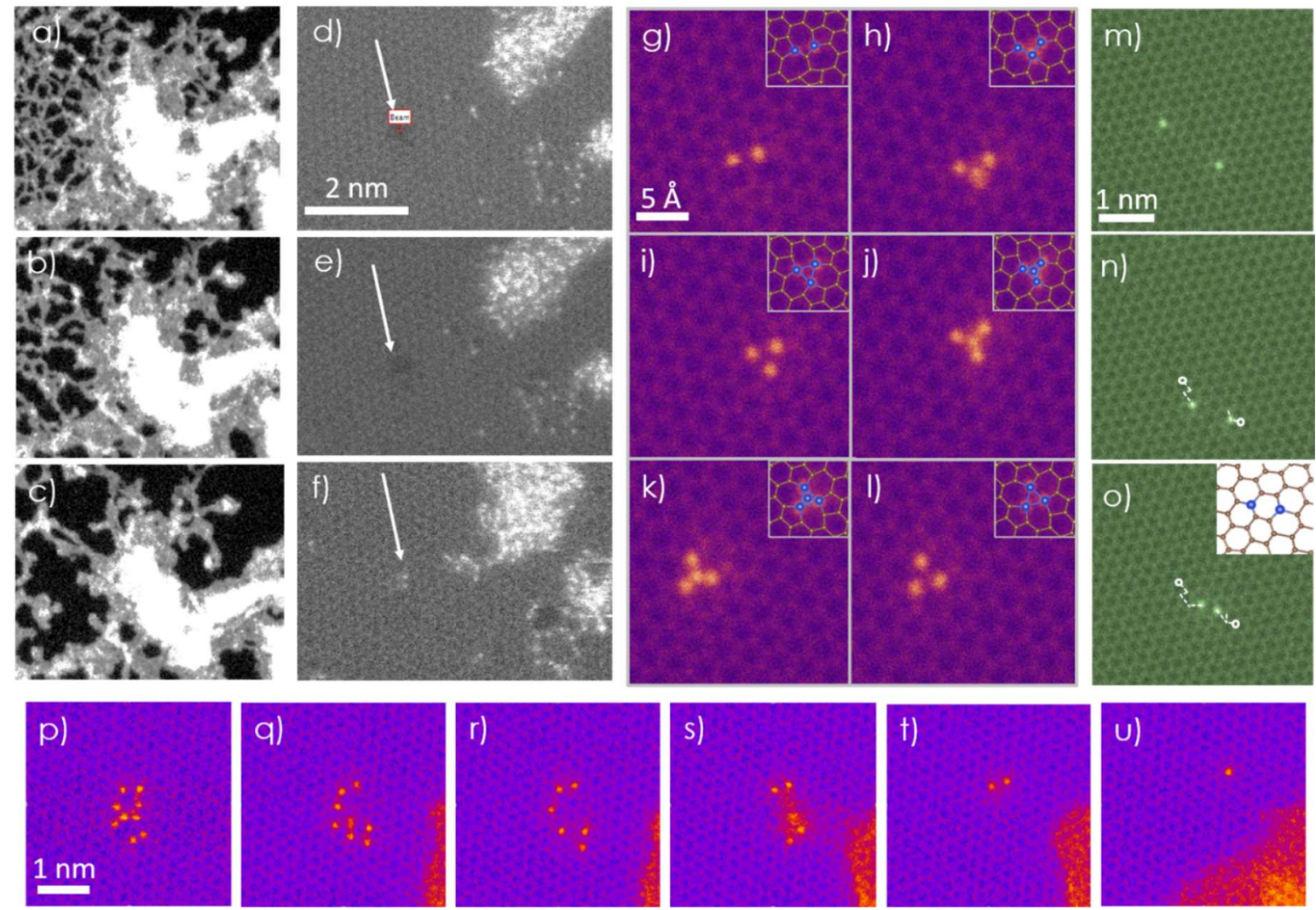

Figure 1. A summary of various beam-induced chemical alterations enabled by tailoring the sample surface environment of graphene. a)-c) illustrate the surface contamination reaction to irradiation by the beam after being chemically treated by $\mathrm{Ar} / \mathrm{O}_{2}$ annealing. The contamination retracts from the graphene revealing large areas of pristine lattice (field of view is $40 \mathrm{~nm}$ wide). d)-f) illustrate the controlled positioning of dopant Si atoms in the graphene lattice. g)-1) show the creation of a trimer and tetramer through beam induced interaction with Si adatoms. m)-o) detail the controlled movement of Si dopants through the graphene lattice to form a dimer. $p$ )-u) show an e-beam induce chemical reaction between ionized hydrocarbon gas and a cluster of Si dopant defects. Beam induced graphene growth is also observed. 